\title{
Models of Relative Nearness Incidence Based on Standard Distance Entropy
}

\author{
Liusan Wu, ${ }^{1,2}$ Qingmei Tan, ${ }^{1}$ and Yuehui Zhang ${ }^{3}$ \\ ${ }^{1}$ School of Economics and Management, Nanjing University of Aeronautics and Astronautics, Nanjing 211106, China \\ ${ }^{2}$ College of Engineering, Nanjing Agricultural University, Nanjing 210031, China \\ ${ }^{3}$ Department of Mathematics, Shanghai Jiao Tong University, Shanghai 200240, China
}

Correspondence should be addressed to Liusan Wu; wuhusheng563520@126.com

Received 19 July 2013; Accepted 17 March 2014; Published 8 April 2014

Academic Editor: Jun Jiang

Copyright (C) 2014 Liusan Wu et al. This is an open access article distributed under the Creative Commons Attribution License, which permits unrestricted use, distribution, and reproduction in any medium, provided the original work is properly cited.

\begin{abstract}
The model of grey nearness incidence cannot reflect the nearness degree of sequences correctly. Therefore, the model of relative nearness incidence of sequences, curves, and surfaces is suggested based on standard distance entropy to remove the current bottlenecks and its properties are studied. At last, three cases are exemplified to demonstrate the validity and practicability of relative nearness incidence. The proposed models have enriched the theory of grey nearness incidence, filled the defects of grey nearness incidence.
\end{abstract}

\section{Introduction}

In 1982, Deng created grey systems theory [1-3], and it has been a far-reaching development in the past 30 years. The subjects of grey systems theory is with part of the information known and part of the information unknown. Therefore, grey systems theory has a very broad prospect of development. In recent years, grey system theory has had successful application in many scientific fields, and it won wide recognition and attention.

As an important part of grey systems theory, grey incidence analysis is the basis of grey forecasting, grey clustering, and grey decision making. The basic idea of grey incidence analysis is based on the curve similarity of different sequences, the closer the curve, the greater the incidence degree and vice versa. Since Deng put forward the theory of grey incidence analysis, many scholars participated in related research, and construct a large number of grey incidence model [4-17]. The models of incidence degree have good applications [18-27]. In addition, scholars study the properties of some incidence degree models $[28,29]$.

Previous studies of the grey incidence model are mainly focused on time sequences. However, there is very few research on the grey incidence model of curves or surfaces.
In addition, many grey incidence models do not satisfy the symmetry. Finally, the grey nearness incidence may be 1 for different sequences; this is obviously unreasonable [12]. Therefore, this paper will construct the grey incidence model of sequences, curves, and surfaces based on the abovementioned defects.

\section{The Model of Grey Nearness Incidence}

With the development of grey incidence theory, Liu et al. proposed the model of grey nearness incidence in 2010; the basic idea is as follows [12].

The system behavior sequence $X_{i}=\left(x_{i}(1), x_{i}(2), \ldots\right.$, $\left.x_{i}(n)\right)$, where $i=1,2, \ldots, t . X_{i}$ and $X_{j}$ denote the crease of $X_{i}$ and $X_{j}$ else. Suppose

$$
S_{i}-S_{j}=\int_{1}^{n}\left(X_{i}-X_{j}\right) d t .
$$

Definition 1. Suppose the length of $X_{i}$ and $X_{j}$ is equal, $S_{i}-S_{j}$ as shown above; then the grey nearness incidence of $X_{i}$ and $X_{j}$ is defined as

$$
\rho_{i j}=\frac{1}{1+\left|S_{i}-S_{j}\right|} .
$$


Theorem 2. Suppose the length of $X_{i}$ and $X_{j}$ is equal; then (notes: part of [12] seems to be missing, now we will correct it)

$$
\begin{aligned}
\rho_{i j}=(1) \times(1 & +\mid \frac{1}{2}\left(x_{i}(1)-x_{j}(1)\right)+\sum_{k=2}^{n-1}\left(x_{i}(k)-x_{j}(k)\right) \\
& \left.+\frac{1}{2}\left(x_{i}(n)-x_{j}(n)\right) \mid\right)^{-1} .
\end{aligned}
$$

However, there are many defects. The grey nearness incidence may be 1 for different sequences. We will use Example 3 to illustrate it.

Example 3. The sequences are as follows:

$$
\begin{gathered}
X_{1}=(1,2,1) \\
X_{2}=(2,1,2) .
\end{gathered}
$$

The corresponding crease of each sequence is shown in Figure 1. We know the grey nearness incidence is $\rho_{12}=1$ based on the above-mentioned model.

This result is clearly not consistent with the fact. So the model of grey nearness incidence cannot reflect the nearness degree of sequences correctly. Therefore, the model of relative nearness incidence of sequences, curves, and surfaces is suggested based on standard distance entropy, and they can solve the above-mentioned problem.

\section{Distance Entropy and Standard Distance Entropy}

Definition 4. Suppose $A$ and $B$ are positive numbers; then the distance entropy of $A$ and $B$ is defined as [30]

$$
H_{A B}=-\left(\frac{A}{A+B} \ln \frac{A}{A+B}+\frac{B}{A+B} \ln \frac{B}{A+B}\right) .
$$
ties:

The distance entropy satisfies the following basic proper-

(1) $H_{A B}=H_{B A}$;

(2) if $A^{*}=x A, B^{*}=x B$, then $H_{A^{*} B^{*}}=H_{A B}$;

(3) $0<H_{A B} \leq \ln 2$ and inf $H=0$;

(4) $H_{A B}=\ln 2$ if and only if $A=B$.

Properties (1) and (2) are obvious; now we prove the properties (3) and (4).

Theorem 5. The maximum value of $f(x)=-[x \ln x+(1-$ $x) \ln (1-x)$ ] on $0<x<1$ is $\ln 2$, the minimum does not exist, and the infimum is 0 .

Proof. Deriving for $f(x)$, we know

$$
f^{\prime}(x)=-[\ln x+1-\ln (1-x)-1]=\ln \left(\frac{1-x}{x}\right) .
$$

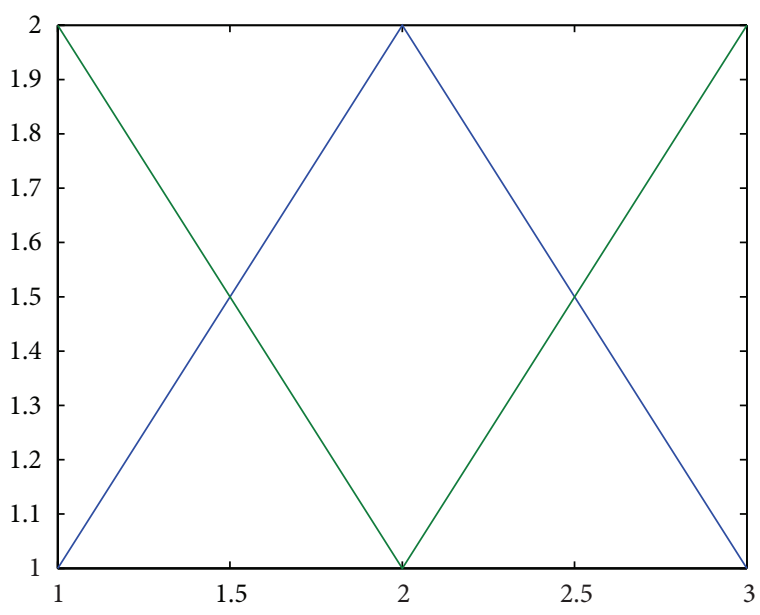

FIGURE 1: The corresponding crease of each sequence.

Suppose $f^{\prime}(x) \geq 0$; then $0<x \leq(1 / 2)$, we know $f(x)$ is an increasing function on $(0,(1 / 2)]$. Suppose $f^{\prime}(x)<$ 0 , then $(1 / 2)<x<1$, we know $f(x)$ is an decreasing function on $((1 / 2), 1)$. Therefore, when $0<x<1, f(x)$ achieves maximum value when $x=(1 / 2)$, and the maximum value is $\max [f(x)]=f(1 / 2)=\ln 2$. Obviously, $f(x)$ has no minimum value in $(0,1)$, and the infimum of $f(x)$ is inf $f(x)=0$. So we obtain the property (3).

During the proof process of Theorem 5, we know the closer the value of $x$ and (1/2), the larger the value of $f(x)$. Therefore, when $A=B>0, H_{A B}$ achieves the maximum value, and the maximum value is $\ln 2$. So we obtain the property (4).

Definition 6. Suppose $A$ and $B$ are positive numbers; then the standard distance entropy of $A$ and $B$ is defined as

$$
\overline{H_{A B}}=\frac{H_{A B}-\inf H_{A B}}{\max H_{A B}-\inf H_{A B}}=\frac{H_{A B}}{\ln 2} .
$$

From the definition of standard distance entropy, we know $0<\overline{H_{A B}} \leq 1$, and the closer the value of $A$ and $B$, the larger the value of $\overline{H_{A B}}$. So we can use $\overline{H_{A B}}$ to express the relative nearness degree of $A$ and $B$.

The standard distance entropy satisfies the following basic properties:

(1) $\overline{H_{A B}}=\overline{H_{B A}}$;

(2) if $A^{*}=x A, B^{*}=x B$, then $\overline{H_{A^{*} B^{*}}}=\overline{H_{A B}}$;

(3) $0<\overline{H_{A B}} \leq 1$;

(4) $\overline{H_{A B}}=1$ if and only if $A=B$.

The properties of standard distance entropy can obtained from the properties of distance entropy, so we will not repeat it. 


\section{The Models of Relative Nearness Incidence Based on Standard Distance Entropy}

4.1. Relative Nearness Incidence of Sequence

Definition 7. For sequences $X_{i}=\left\{x_{i}(1), x_{i}(2), \ldots, x_{i}(n)\right\}$, where $x_{i}(t)>0, i=1,2, \ldots, m, t=1,2, \ldots, n$. Then the distance entropy of $x_{i}(t)$ and $x_{j}(t)$ is defined as

$$
\begin{aligned}
H_{i j}(t)=-( & \frac{x_{i}(t)}{x_{i}(t)+x_{j}(t)} \ln \frac{x_{i}(t)}{x_{i}(t)+x_{j}(t)} \\
& \left.+\frac{x_{j}(t)}{x_{i}(t)+x_{j}(t)} \ln \frac{x_{j}(t)}{x_{i}(t)+x_{j}(t)}\right) .
\end{aligned}
$$

Standardising $H_{i j}(t)$, we know

$$
\overline{H_{i j}(t)}=\frac{H_{i j}(t)}{\ln 2} .
$$

$\overline{H_{i j}(t)}$ can express the relative nearness degree of $x_{i}(t)$ and $x_{j}(t)$; then the average of $\overline{H_{i j}(t)}$ can express the relative nearness incidence of $X_{i}$ and $X_{j}$. So we have Theorem 8 .

Theorem 8. Sequences $X_{i}=\left\{x_{i}(1), x_{i}(2), \ldots, x_{i}(n)\right\}$ and $X_{j}=$ $\left\{x_{j}(1), x_{j}(2), \ldots, x_{j}(n)\right\}$ are constant positive; then the relative nearness incidence of $X_{i}$ and $X_{j}$ is

$$
\begin{aligned}
& H_{i j}=\frac{1}{n} \sum_{t=1}^{n} \overline{H_{i j}(t)}=\frac{1}{n \ln 2} \sum_{t=1}^{n} H_{i j}(t) \\
&=-\frac{1}{n \ln 2} \sum_{t=1}^{n}\left(\frac{x_{i}(t)}{x_{i}(t)+x_{j}(t)} \ln \frac{x_{i}(t)}{x_{i}(t)+x_{j}(t)}\right. \\
&\left.+\frac{x_{j}(t)}{x_{i}(t)+x_{j}(t)} \ln \frac{x_{j}(t)}{x_{i}(t)+x_{j}(t)}\right),
\end{aligned}
$$

where $\overline{H_{i j}(t)}$ means the standard distance entropy of $x_{i}(t)$ and $x_{j}(t)$.

\subsection{Relative Nearness Incidence of Curve}

Definition 9. For curves $y_{i}=f_{i}(x)$ and $y_{j}=f_{j}(x)$, where $y_{i}=$ $f_{i}(x)>0$ and $y_{j}=f_{j}(x)>0$ on $x \in[a, b], i=1,2, \ldots, m$, when $x=x_{0}$, the distance entropy of $y_{i}=f_{i}(x)$ and $y_{j}=$ $f_{j}(x)$ is defined as

$$
\begin{aligned}
H_{i j}\left(x_{0}\right)=-( & \frac{f_{i}\left(x_{0}\right)}{f_{i}\left(x_{0}\right)+f_{j}\left(x_{0}\right)} \ln \frac{f_{i}\left(x_{0}\right)}{f_{i}\left(x_{0}\right)+f_{j}\left(x_{0}\right)} \\
& \left.+\frac{f_{j}\left(x_{0}\right)}{f_{i}\left(x_{0}\right)+f_{j}\left(x_{0}\right)} \ln \frac{f_{j}\left(x_{0}\right)}{f_{i}\left(x_{0}\right)+f_{j}\left(x_{0}\right)}\right) .
\end{aligned}
$$

Standardising $H_{i j}(t)$, we know

$$
\overline{H_{i j}\left(x_{0}\right)}=\frac{H_{i j}\left(x_{0}\right)}{\ln 2} .
$$

$\overline{H_{i j}\left(x_{0}\right)}$ can express the relative nearness degree of $y_{i}=f_{i}(x)$ and $y_{j}=f_{j}(x)$ when $x=x_{0}$. So we have Theorem 10 .
Theorem 10. Curves $y_{i}=f_{i}(x)$ and $y_{j}=f_{j}(x)$ and they are positive on $x \in[a, b]$; then the relative nearness incidence of $y_{i}=f_{i}(x)$ and $y_{j}=f_{j}(x)$ is

$$
\begin{aligned}
H_{i j}(x) & \\
= & \frac{1}{b-a} \int_{a}^{b} \overline{H_{i j}\left(x_{0}\right)} d x_{0} \\
= & -\frac{1}{(b-a) \ln 2} \int_{a}^{b} H_{i j}\left(x_{0}\right) d x_{0} \\
= & -\frac{1}{(b-a) \ln 2} \\
& \times \int_{a}^{b}\left(\frac{f_{i}\left(x_{0}\right)}{f_{i}\left(x_{0}\right)+f_{j}\left(x_{0}\right)} \ln \frac{f_{i}\left(x_{0}\right)}{f_{i}\left(x_{0}\right)+f_{j}\left(x_{0}\right)}\right. \\
& \left.\quad+\frac{f_{j}\left(x_{0}\right)}{f_{i}\left(x_{0}\right)+f_{j}\left(x_{0}\right)} \ln \frac{f_{j}\left(x_{0}\right)}{f_{i}\left(x_{0}\right)+f_{j}\left(x_{0}\right)}\right) d x_{0} .
\end{aligned}
$$

\subsection{Relative Nearness Incidence of Surface}

Definition 11. For surfaces $z_{i}=f_{i}(x, y)$ and $z_{j}=f_{j}(x, y)$, where $z_{i}=f_{i}(x, y)>0$ and $z_{j}=f_{j}(x, y)>0$ on $x \in[a, b]$ and $y \in[c, d], i=1,2, \ldots, m$, when $x=x_{0}$ and $y=y_{0}$, the distance entropy of $z_{i}=f_{i}(x, y)$ and $z_{j}=f_{j}(x, y)$ is defined as

$$
\begin{aligned}
& H_{i j}\left(x_{0}, y_{0}\right) \\
& =-\left(\frac{f_{i}\left(x_{0}, y_{0}\right)}{f_{i}\left(x_{0}, y_{0}\right)+f_{j}\left(x_{0}, y_{0}\right)} \ln \frac{f_{i}\left(x_{0}, y_{0}\right)}{f_{i}\left(x_{0}, y_{0}\right)+f_{j}\left(x_{0}, y_{0}\right)}\right. \\
& \quad+\frac{f_{j}\left(x_{0}, y_{0}\right)}{f_{i}\left(x_{0}, y_{0}\right)+f_{j}\left(x_{0}, y_{0}\right)} \\
& \left.\quad \times \ln \frac{f_{j}\left(x_{0}, y_{0}\right)}{f_{i}\left(x_{0}, y_{0}\right)+f_{j}\left(x_{0}, y_{0}\right)}\right) .
\end{aligned}
$$

Standardising $H_{i j}\left(x_{0}, y_{0}\right)$, we know

$$
\overline{H_{i j}\left(x_{0}, y_{0}\right)}=\frac{H_{i j}\left(x_{0}, y_{0}\right)}{\ln 2}
$$

$\overline{H_{i j}\left(x_{0}, y_{0}\right)}$ can express the relative nearness degree of $z_{i}=$ $f_{i}(x, y)$ and $z_{j}=f_{j}(x, y)$ when $x=x_{0}$ and $y=y_{0}$. So we have Theorem 12 
Theorem 12. Surfaces $z_{i}=f_{i}(x, y)$ and $z_{j}=f_{j}(x, y)$ and they are positive on $x \in[a, b], y \in[c, d]$. Then the relative nearness incidence of $z_{i}=f_{i}(x, y)$ and $z_{j}=f_{j}(x, y)$ is

$$
\begin{aligned}
& H_{i j}(x, y) \\
& =\frac{1}{(b-a)(d-c)} \int_{c}^{d} \int_{a}^{b} \overline{H_{i j}\left(x_{0}, y_{0}\right)} d x_{0} d y_{0} \\
& =-\frac{1}{(b-a)(d-c) \ln 2} \int_{c}^{d} \int_{a}^{b} H_{i j}\left(x_{0}, y_{0}\right) d x_{0} d y_{0} \\
& =-\frac{1}{(b-a)(d-c) \ln 2} \\
& \quad \times \int_{c}^{d} \int_{a}^{b}\left(\frac{f_{i}\left(x_{0}, y_{0}\right)}{f_{i}\left(x_{0}, y_{0}\right)+f_{j}\left(x_{0}, y_{0}\right)}\right. \\
& \quad \times \ln \frac{f_{i}\left(x_{0}, y_{0}\right)}{f_{i}\left(x_{0}, y_{0}\right)+f_{j}\left(x_{0}, y_{0}\right)} \\
& \quad+\frac{f_{j}\left(x_{0}, y_{0}\right)}{f_{i}\left(x_{0}, y_{0}\right)+f_{j}\left(x_{0}, y_{0}\right)} \\
& \left.\quad \times \ln \frac{f_{j}\left(x_{0}, y_{0}\right)}{f_{i}\left(x_{0}, y_{0}\right)+f_{j}\left(x_{0}, y_{0}\right)}\right) d x_{0} d y_{0} .
\end{aligned}
$$

Theorem 13. The relative nearness incidence of sequences, curves, and surfaces satisfies the following properties:

(1) $0<H_{i j} \leq 1,0<H_{i j}(x) \leq 1,0<H_{i j}(x, y) \leq 1$;

(2) $H_{i j}=H_{j i}, H_{i j}(x)=H_{j i}(x), H_{i j}(x, y)=H_{j i}(x, y)$;

(3) $H_{i j}=1$ if and only if $x_{i}(t)=x_{j}(t) ; H_{i j}(x)=1$ if and only if $f_{i}(x)=f_{j}(x) ; H_{i j}(x, y)=1$ if and only if $f_{i}(x, y)=f_{j}(x, y)$;

(4) if $X_{i}^{\prime}=a X_{i}, X_{j}^{\prime}=a X_{j}$, then $H_{i j}^{\prime}=H_{i j}$; if $f_{i}^{\prime}(x)=$ $a f_{i}(x), f_{j}^{\prime}(x)=a f_{j}(x), H_{i j}^{\prime}(x)=H_{i j}(x)$; if $f_{i}^{\prime}(x, y)=$ $a f_{i}(x, y), f_{j}^{\prime}(x, y)=a f_{j}(x, y)$, then $H_{i j}^{\prime}(x, y)=$ $H_{i j}(x, y)$.

The above-mentioned properties are obvious, so we will not repeat them here.

\section{Examples}

In order to illustrate the validity and practicability of the relative nearness incidence, we have the following 3 examples.

Example 1. Suppose

$$
\begin{aligned}
& X_{1}=(0.5,0.9,1.2,2.0) \\
& X_{2}=(0.4,0.5,1.6,2.3) \\
& X_{3}=(0.6,0.9,1.8,2.2) .
\end{aligned}
$$

As seen in the above, we know

$$
\begin{array}{ll}
H_{12}(1)=0.6870, & H_{12}(2)=0.6518, \\
H_{12}(3)=0.6829, & H_{12}(4)=0.6907, \\
H_{13}(1)=0.6890, & H_{13}(2)=\ln 2, \\
H_{13}(3)=0.6730, & H_{13}(4)=0.6920 .
\end{array}
$$

So

$$
\begin{aligned}
& H_{12}=\frac{1}{4 \ln 2}(0.6870+0.6518+0.6829+0.6907)=0.9783 \\
& H_{13}=\frac{1}{4 \ln 2}(0.6890+\ln 2+0.6730+0.6920)=0.9908 .
\end{aligned}
$$

Then

$$
H_{12}<H_{13} .
$$

So the relative nearness incidence of $X_{1}$ and $X_{2}$ is weaker than the relative nearness incidence of $X_{1}$ and $X_{3}$.

In order to compare the relative nearness incidence of sequences with the relative degree of grey incidence of sequences, we put the data of Example 2 into the formula of relative degree of grey incidences. It is easy to know that $\gamma_{12}=0.8425$ and $\gamma_{13}=0.9844$. So

$$
\gamma_{12}<\gamma_{13} .
$$

Then the relative degree of grey incidences of $X_{1}$ and $X_{2}$ is weaker than the relative degree of grey incidences of $X_{1}$ and $X_{3}$. Therefore, the relative nearness incidence of sequences and the relative degree of grey incidences of sequences are harmonious.

Example 2. Suppose $f_{1}(x)=x, f_{2}(x)=x^{2}, f_{3}(x)=x^{3}$, where $x \in[0.5,2]$. As seen in the above, we know

$$
\begin{aligned}
& H_{12}\left(x_{0}\right)=-\left(\frac{x_{0}}{x_{0}+x_{0}^{2}} \ln \frac{x_{0}}{x_{0}+x_{0}^{2}}+\frac{x_{0}^{2}}{x_{0}+x_{0}^{2}} \ln \frac{x_{0}^{2}}{x_{0}+x_{0}^{2}}\right) \\
& H_{13}\left(x_{0}\right)=-\left(\frac{x_{0}}{x_{0}+x_{0}^{3}} \ln \frac{x_{0}}{x_{0}+x_{0}^{3}}+\frac{x_{0}^{3}}{x_{0}+x_{0}^{3}} \ln \frac{x_{0}^{3}}{x_{0}+x_{0}^{3}}\right) .
\end{aligned}
$$

Then

$$
\begin{aligned}
& H_{12}(x) \\
& =\frac{1}{(2-0.5) \ln 2} \int_{0.5}^{2} H_{12}\left(x_{0}\right) d x_{0} \\
& =-\frac{1}{(2-0.5) \ln 2} \\
& \quad \times \int_{0.5}^{2}\left(\frac{x_{0}}{x_{0}+x_{0}^{2}} \ln \frac{x_{0}}{x_{0}+x_{0}^{2}}+\frac{x_{0}^{2}}{x_{0}+x_{0}^{2}} \ln \frac{x_{0}^{2}}{x_{0}+x_{0}^{2}}\right) d x_{0}
\end{aligned}
$$

$=0.9705$ 


$$
\begin{aligned}
& H_{13}(x) \\
&= \frac{1}{(2-0.5) \ln 2} \int_{0.5}^{2} H_{13}\left(x_{0}\right) d x_{0} \\
&=-\frac{1}{(2-0.5) \ln 2} \\
& \times \int_{0.5}^{2}\left(\frac{x_{0}}{x_{0}+x_{0}^{3}} \ln \frac{x_{0}}{x_{0}+x_{0}^{3}}+\frac{x_{0}^{3}}{x_{0}+x_{0}^{3}} \ln \frac{x_{0}^{3}}{x_{0}+x_{0}^{3}}\right) d x_{0} \\
&= 0.8931 .
\end{aligned}
$$

Obviously, when $x \in[0.5,2]$, then $H_{12}(x)>H_{13}(x)$. So the relative nearness incidence of $f_{1}(x)$ and $f_{2}(x)$ is stronger than the relative nearness incidence of $f_{1}(x)$ and $f_{3}(x)$.

Example 3. Suppose $f_{1}(x, y)=x^{2}+y, f_{2}(x, y)=x+y$, $f_{3}(x, y)=x^{2}+2 y^{2}$, where $x \in[0.3,1.5], y \in[0.2,1.3]$. As seen in the above, we know

$$
\begin{gathered}
H_{12}\left(x_{0}, y_{0}\right) \\
=-\left(\frac{x_{0}^{2}+y_{0}}{\left(x_{0}^{2}+y_{0}\right)+\left(x_{0}+y_{0}\right)} \ln \frac{x_{0}^{2}+y_{0}}{\left(x_{0}^{2}+y_{0}\right)+\left(x_{0}+y_{0}\right)}\right. \\
\left.\quad+\frac{x_{0}+y_{0}}{\left(x_{0}^{2}+y_{0}\right)+\left(x_{0}+y_{0}\right)} \ln \frac{x_{0}+y_{0}}{\left(x_{0}^{2}+y_{0}\right)+\left(x_{0}+y_{0}\right)}\right) \\
H_{13}\left(x_{0}, y_{0}\right) \\
=-\left(\frac{x_{0}^{2}+y_{0}}{\left(x_{0}^{2}+y_{0}\right)+\left(x_{0}^{2}+2 y_{0}^{2}\right)} \ln \frac{x_{0}^{2}+y_{0}}{\left(x_{0}^{2}+y_{0}\right)+\left(x_{0}^{2}+2 y_{0}^{2}\right)}\right. \\
\left.\quad+\frac{x_{0}^{2}+2 y_{0}^{2}}{\left(x_{0}^{2}+y_{0}\right)+\left(x_{0}^{2}+2 y_{0}^{2}\right)} \ln \frac{x_{0}^{2}+2 y_{0}^{2}}{\left(x_{0}^{2}+y_{0}\right)+\left(x_{0}^{2}+2 y_{0}^{2}\right)}\right) .
\end{gathered}
$$

Then

$$
\begin{aligned}
& H_{12}(x, y) \\
& =\frac{1}{(1.5-0.3)(1.3-0.2) \ln 2} \\
& \quad \times \int_{0.2}^{1.3} \int_{0.3}^{1.5} H_{12}\left(x_{0}, y_{0}\right) d x_{0} d y_{0} \\
& =-\frac{1}{(1.5-0.3)(1.3-0.2) \ln 2} \\
& \times \int_{0.2}^{1.3} \int_{0.3}^{1.5}\left(\frac{x_{0}^{2}+y_{0}}{\left(x_{0}^{2}+y_{0}\right)+\left(x_{0}+y_{0}\right)}\right. \\
& \quad \times \ln \frac{x_{0}^{2}+y_{0}}{\left(x_{0}^{2}+y_{0}\right)+\left(x_{0}+y_{0}\right)} \\
& \quad \times \frac{x_{0}+y_{0}}{\left(x_{0}^{2}+y_{0}\right)+\left(x_{0}+y_{0}\right)} \\
& \left.\quad \frac{x_{0}+y_{0}}{\left(x_{0}^{2}+y_{0}\right)+\left(x_{0}+y_{0}\right)}\right) d x_{0} d y_{0}
\end{aligned}
$$

$=0.9937$

$$
\begin{aligned}
& H_{13}(x, y) \\
& =\frac{1}{(1.5-0.3)(1.3-0.2) \ln 2} \\
& \times \int_{0.2}^{1.3} \int_{0.3}^{1.5} H_{13}\left(x_{0}, y_{0}\right) d x_{0} d y_{0} \\
& =-\frac{1}{(1.5-0.3)(1.3-0.2) \ln 2} \\
& \times \int_{0.2}^{1.3} \int_{0.3}^{1.5}\left(\frac{x_{0}^{2}+y_{0}}{\left(x_{0}^{2}+y_{0}\right)+\left(x_{0}^{2}+2 y_{0}^{2}\right)}\right. \\
& \times \ln \frac{x_{0}^{2}+y_{0}}{\left(x_{0}^{2}+y_{0}\right)+\left(x_{0}^{2}+2 y_{0}^{2}\right)} \\
& +\frac{x_{0}^{2}+2 y_{0}^{2}}{\left(x_{0}^{2}+y_{0}\right)+\left(x_{0}^{2}+2 y_{0}^{2}\right)} \\
& \left.\times \ln \frac{x_{0}^{2}+2 y_{0}^{2}}{\left(x_{0}^{2}+y_{0}\right)+\left(x_{0}^{2}+2 y_{0}^{2}\right)}\right) d x_{0} d y_{0}
\end{aligned}
$$

$=0.9769$.

Obviously, when $x \in[0.3,1.5]$ and $y \in[0.2,1.3]$, then $H_{12}(x, y)>H_{13}(x, y)$. So the relative nearness incidence of $f_{1}(x, y)$ and $f_{2}(x, y)$ is stronger than the relative nearness incidence of $f_{1}(x, y)$ and $f_{3}(x, y)$.

\section{Conclusion}

The model of grey nearness incidence proposed by Liu et al. cannot reflect the nearness degree of sequences correctly. Therefore, the model of relative nearness incidence of sequences, curves, and surfaces is suggested based on the standard distance entropy to remove the current bottlenecks. The proposed models have enriched the theory of grey nearness incidence, filled the defects of grey nearness incidence. However, only two positive numbers can calculate its standard distance entropy. Therefore, there are many defects of relative nearness incidence based on standard distance entropy; our future research will focus on it.

\section{Conflict of Interests}

The authors declare that there is no conflict of interests regarding the publication of this paper.

\section{Acknowledgments}

The authors are grateful to the editor and anonymous reviewers for their valuable suggestions which improved the paper. This paper was partially supported by the National Natural Science Foundation of China (nos. 71073079 and 11271257); the National Social Science Foundation of China (no. 12BGL104); the Funding of Jiangsu Innovation Program for Graduate Education (no. CXLX12_0174); and the Fundamental Research Funds for the Central Universities (no. NC2012009). 


\section{References}

[1] J. L. Deng, The Primary Methods of Grey Systems Theory, Huazhong University of Science and Technology, Wuhan, China, 1987.

[2] J. L. Deng, "Grey incidence space in grey systems theory," Fuzzy Mathematics, vol. 4, no. 2, pp. 1-10, 1985.

[3] J. L. Deng, "Grey information space," The Journal of Grey System, vol. 1, no. 2, pp. 103-117, 1989.

[4] Z. G. Mei, "The concept and computation method of grey bbsolute correlation degree," Systems Engineering, vol. 5, no. 8, pp. 15-18, 1992.

[5] S. F. Liu, Y. G. Dang, Z. G. Fang et al., Grey System Theory and Its Applications, Science Press, Beijing, China, 5th edition, 2010.

[6] Q. Y. Wang, "On grey incidence analysis model of type B," Journal of Huazhong University of Science and Technology, vol. 17, no. 6, pp. 77-82, 1989.

[7] Q. Y. Wang and X. H. Zhao, "The relational analysis of C-mode," Journal of Huazhong University of Science and Technology, vol. 27, no. 3, pp. 75-77, 1999.

[8] W. X. Tang, "On grey incidence degree model of type T with its calculation," Mathematical Statitics and Management, vol. 14, no. 1, pp. 33-37, 1995.

[9] S. F. Liu, Z. G. Fang, and Y. Lin, "Study on a new definition of grey incidence," The Journal of Grey System, vol. 9, no. 2, pp. 115122, 2006.

[10] Y. L. Zhao, S. Y. Wei, and Z. X. Mei, "Grey euclid relation grade," Journal of Guangxi University (Natural Science Edition), vol. 23, no. 1, pp. 10-13, 1998.

[11] Y. G. Dang, S. F. Liu, B. Liu et al., "Improvement on degree ofgrey slope incidence," Engineering Science, vol. 6, no. 3, pp. 41-44, 2004.

[12] S. Liu, N. Xie, and J. Forrest, "On new models of grey incidence analysis based on visual angle of similarity and nearness," System Engineering Theory and Practice, vol. 30, no. 5, pp. 881$887,2010$.

[13] S. F. Liu and Y. Lin, An Introduction to Grey System: Foundations, Methodology and Applications, IIGSS Academic Publisher, New York, NY, USA, 1998.

[14] L. F. Wu, Y. N. Wang, and S. F. Liu, "Grey convex relation and its properties," Systems Engineering-Theory and Practice, no. 7, pp. 1501-1505, 2012.

[15] H. Shi, S. Liu, Z. Fang, and B. Yang, "Grey amplitude incidence model," System Engineering Theory and Practice, vol. 30, no. 10, pp. 1828-1833, 2010.

[16] B. Zeng, S. F. Liu, W. Meng et al., "Incidence degree model of interval grey number based on space mapping," Systems Engineering, vol. 28, no. 8, pp. 122-126, 2010.

[17] K. Zhang and S. F. Liu, "A novel algorithm of image edge detection based on matrix degree of grey incidences," The Journal of Grey System, vol. 19, no. 3, pp. 265-276, 2009.

[18] J. Wang, S. Liu, and M. Liu, "Grey relational analysis models with incomplete information based on cross-evaluation," System Engineering Theory and Practice, vol. 30, no. 4, pp. 732-737, 2010.

[19] H. Ke, S. Liu, Y. Chen, and Z. Fang, "New solution algorithm for multiple objective programming model based on grey relational degree," Systems Engineering and Electronics, vol. 32, no. 3, pp. 544-547, 2010.

[20] K. Zhang, W. Ye, and L. Zhao, "The absolute degree of grey incidence for grey sequence base on standard grey interval number operation," Kybernetes, vol. 41, no. 7-8, pp. 934-944, 2012.

[21] J. Cui, Y. G. Dang, and S. F. Liu, "An improved approach for determining weights of attributes in decision making based on grey incidence," Chinese Journal of Management Science, vol. 16, no. 5, pp. 141-145, 2008.

[22] Y. Wang, "Analysis of FDI and Chinese regional economic discrepancy using grey incidence theory," System Engineering Theory and Practice, vol. 30, no. 3, pp. 426-430, 2010.

[23] G. Q. Yu, Z. L. Wang, and B. S. Zhang, "The grey relational analysis of sluice monitoring data," Procedia Engineering, vol. 15, pp. 5192-5196, 2011.

[24] B. Yang, Z. Fang, W. Zhou, and J. Liu, "Incidence decision model of multi-attribute interval grey number based on information reduction operator," Control and Decision, vol. 27, no. 2, pp. 182186, 2012.

[25] F. Dong, Q. M. Tan, D. Q. Zhou et al., "The effect of technological progress, industrial structure and opening-up degree on Chinese energy consumption: an empirical study based on the two-step method, the grey relational analysis and co-integration test," China Population, Resources and Environment, vol. 20, no. 6, pp. 22-27, 2010.

[26] Y. Q. Guan and D. J. Song, "Correlation analysis of bearing track using grey system theory," Kybernetes, vol. 41, pp. 7945-8952, 2012.

[27] L. P. Jiang, "Gas path fault diagnosis system of aero-engine based on Grey Relationship degree," Procedia Engineering, vol. 15, pp. 4774-4779, 2011.

[28] N. M. Xie and S. F. Liu, "The parallel and uniform properties of several relational models," Systems Engineering, vol. 25, no. 8, pp. 98-103, 2007.

[29] J. Cui, Y. G. Dang, and S. F. Liu, "Novel properties of some grey relational analysis models," Systems Engineering, vol. 27, no. 4, pp. 65-70, 2009.

[30] P. F. Wang, The Study of Uncerten Multiple Attribute DecisionMaking Problems Based on the Grey Entropy, Nanjing University of Aeronautics and Astronautics, Nanjing, China, 2009. 


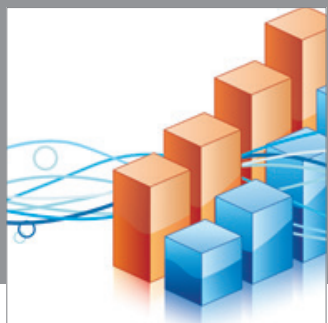

Advances in

Operations Research

mansans

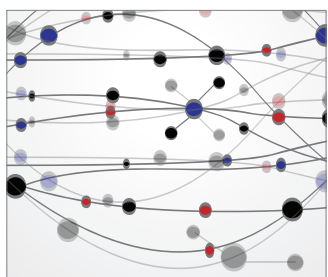

The Scientific World Journal
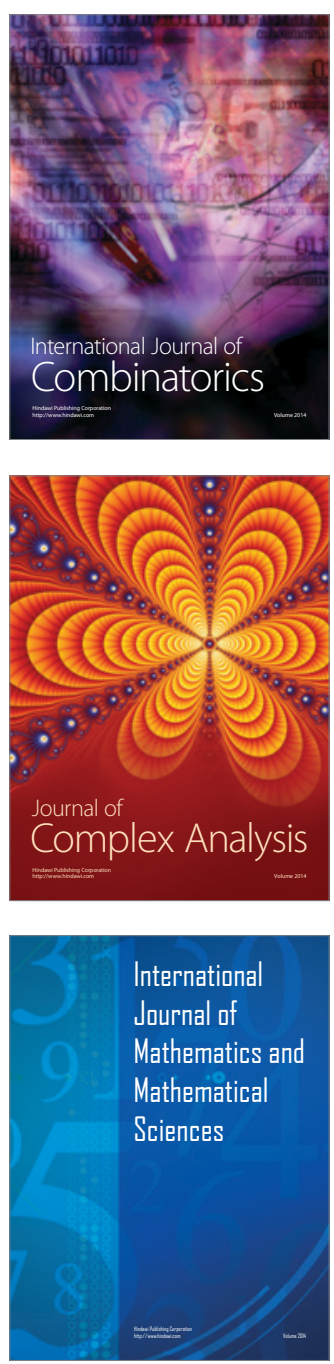
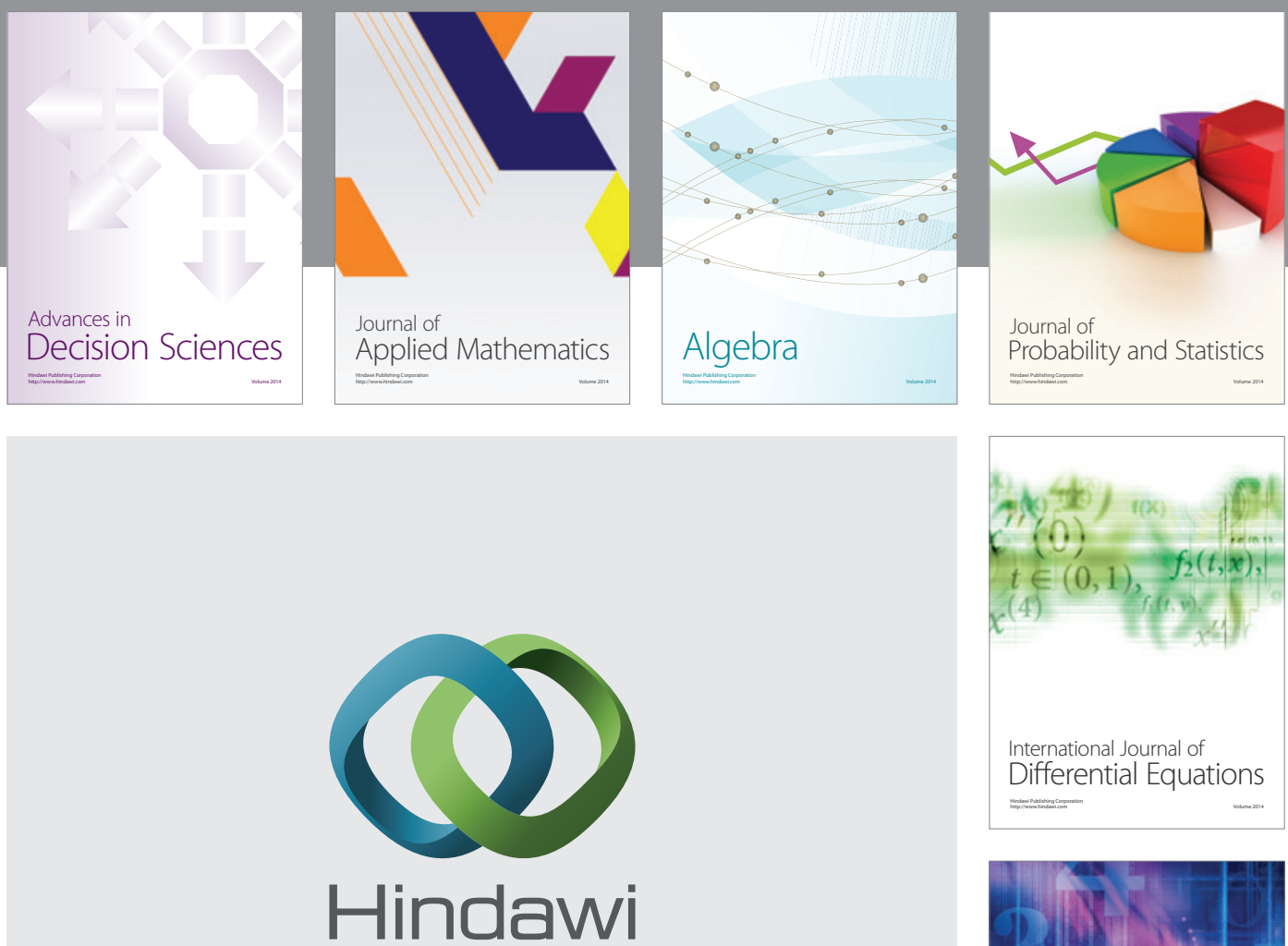

Submit your manuscripts at http://www.hindawi.com
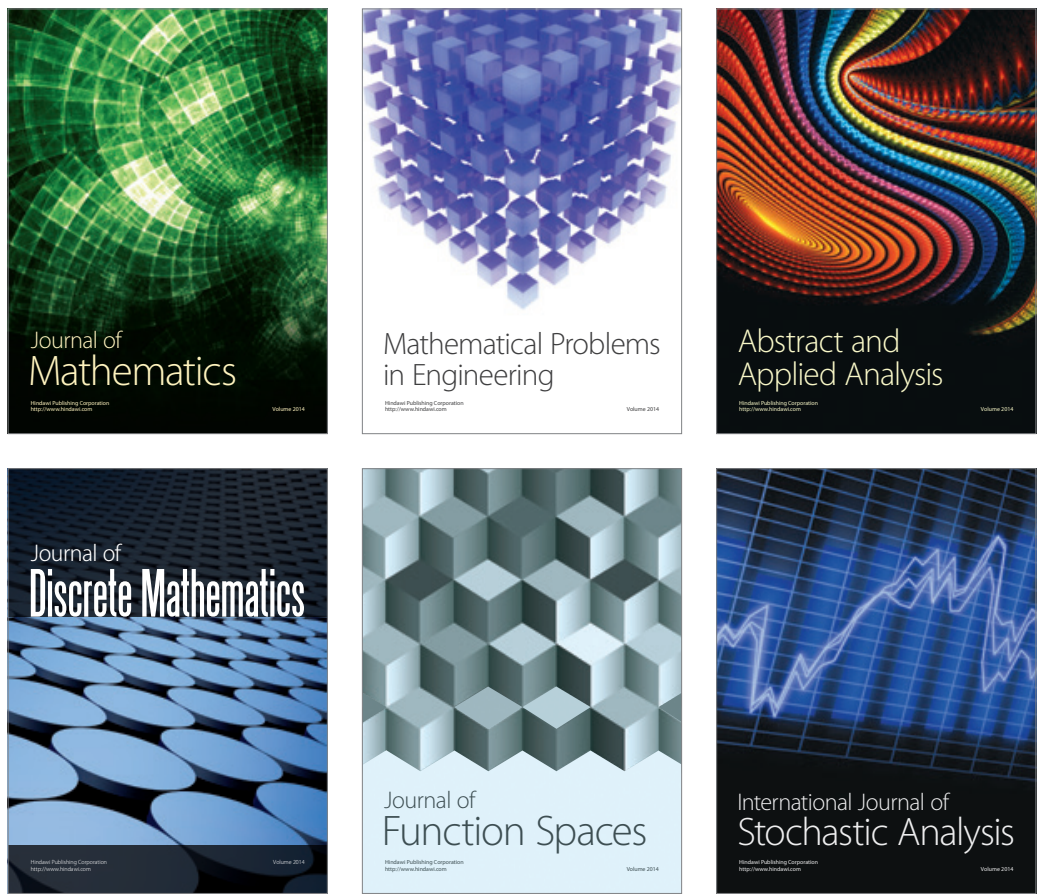

Journal of

Function Spaces

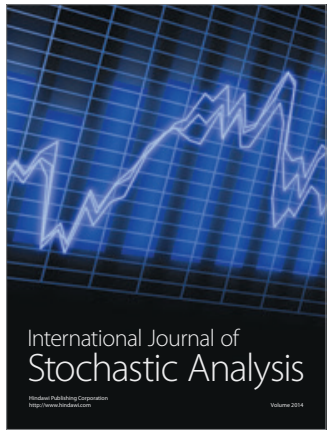

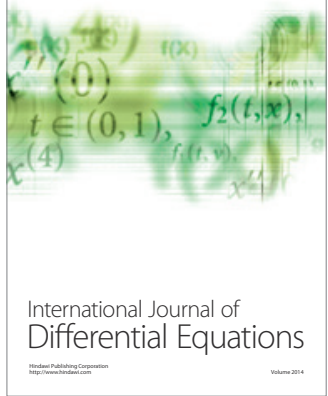
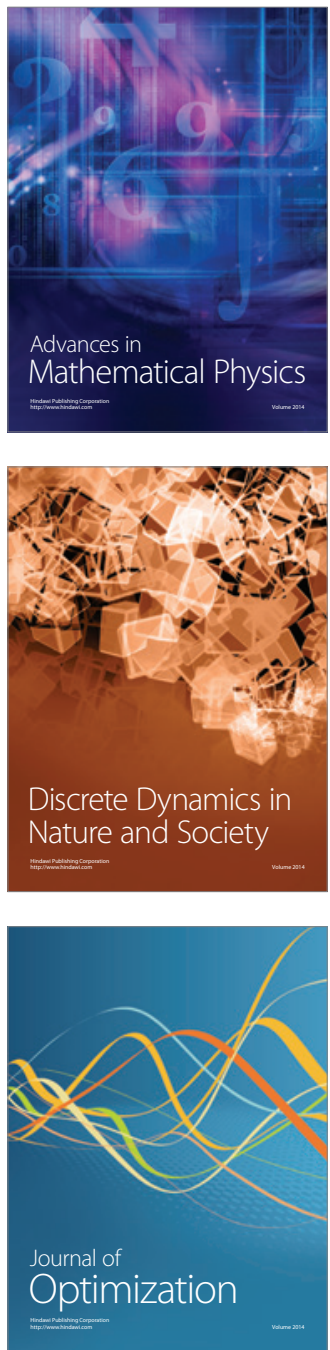